\title{
TAXUS ATLAS
}

\section{Evaluation of TAXUS Liberté versus TAXUS Express Stent Evaluation des TAXUS Liberté im Vergleich zum TAXUS-Express-Stent}

Hintergrund und Problemstellung: Der TAXUS-ExpressStent setzt aus einer Polymerbeschichtung Paclitaxel frei. In zahlreichen klinischen, kontrollierten, randomisierten Studien (TAXUS-I, -II, -IV, -V und -VI) wurden seine Wirksamkeit und Sicherheit in Koronarstenosen unterschiedlicher Komplexität erfolgreich dokumentiert. Später wurde das Stentdesign durch dünnere Stentstreben und eine kontinuierliche Zellarchitektur mit höherer Flexibilität und einem niedrigeren Profil verbessert (TAXUS Liberté). Um nicht

\begin{tabular}{|c|c|}
\hline Methodik & \\
\hline Studiendesign & $\begin{array}{l}\text { Kontrollierte Registerstudie mit Powerkalkula- } \\
\text { tion }\end{array}$ \\
\hline Primärer Endpunkt & TVR nach 9 Monaten (non-inferior) \\
\hline Sekundäre Endpunkte & Die von TAXUS-IV und TAXUS-V \\
\hline Patientenauswahl & $\begin{array}{l}\text { Einschlusskriterien: } \\
\text { De-novo-Koronarstenosen von 10-28 mm Län- } \\
\text { ge, Referenzgefäßdurchmesser: 2,5-4,0 mm, } \\
\text { mit einem einzigen Stent behandelbar }\end{array}$ \\
\hline & $\begin{array}{l}\text { Ausschlusskriterien: } \\
\text { Die von TAXUS-IV und TAXUS-V }\end{array}$ \\
\hline
\end{tabular}

Anzahl der teilnehmenden Zentren 61

Evidence-based-Medicine- (EBM-) Score (www.TCTMD.com)

Klinischer primärer Endpunkt Doppelblind (einschließlich Ärzten)

Beobachtungsintervall für den primären Endpunkt $\geq 6$ Monate

Multicenter (mindestens 3 Zentren)

Externes und vom Steering Comittee unabhängiges Clinical Event Committee/DSMB

(Datensicherheit Monitoring Board)

Primärer Endpunkt erreicht

Power von $\geq 80 \%$ für den primären Endpunkt erreicht

Anteil des Follow-up der Patienten für angiographischen primären Endpunkt $\geq 80 \%$ und $\geq 95 \%$ für klinischen primären

Endpunkt

\section{Gesamt-EBM-Score}

8

"Silber Score" für randomisierte, kontrollierte Studien (RaCT) oder kontrollierte Registerstudien (ReCT mit präspezifizierter Kontrollgruppe und Power Kalkulation); es sind maximal 10 Scorepunkte möglich.

Schlussfolgerung und Kommentar: TAXUS ATLAS ist eine qualitativ hochwertige Registerstudie, da sie auf der Basis einer präspezifizierten Kontrollgruppe mit Einschluss- und Ausschlusskriterien auf dem Boden einer Power-Kalkulation eine Patientenanzahl berechnete. Aus diesem Grunde kann bei einer solchen Registerstudie durchaus von einem „echten primären Endpunkt" gesprochen werden. Der primäre Endpunkt (TAXUS Liberté ist dem TAXUS Express nicht unterlegen) wurde erreicht, umso mehr, als in der TAXUS-Liberté-Gruppe Patienten mit komplexeren Stenosen enthalten sämtliche TAXUS-Studien mit dem TAXUS-Liberté-Stent zu wiederholen, wurde TAXUS ATLAS als prospektive, kon-trollierte Registerstudie konzipiert: Anhand einer präspezifizierten historischen Kontrollgruppe der TAXUS-IVund -V-Studien wurden eine Power-Kalkulation in Analogie zu einer randomisierten, kontrollierten Studie durchgeführt und hieraus die notwendige Patientenanzahl für diese „noninferiority"-Registerstudie berechnet. Studienleiter war M.A. Turco, Takoma Park, USA.

\section{Ergebnisse $\left({ }^{*} p<0,05\right)$}

\begin{tabular}{|c|c|}
\hline & TAXUS Liberté \\
\hline Patientenanzahl & $\mathrm{n}=991$ \\
\hline Patientencharakteristika & $\begin{array}{l}\text { Die Patientengruppen waren hinsichtlich } \\
\text { des Referenzgefäßdurchmessers vergleich- } \\
\text { bar }(2,79 \pm 0,49 \text { vs. } 2,75 \pm 0,50 \mathrm{~mm}) \text {. } \\
\text { Auch die Anzahl der Stents pro Läsion war } \\
\text { identisch (jeweils } 1,1 \pm 0,3 \mathrm{~mm}) \text {. Multi- } \\
\text { ple Stents wurden in } 7,9 \text { bzw. } 7,5 \% \text { der } \\
\text { Patienten eingesetzt. Signifikante Unter- } \\
\text { schiede zwischen beiden Gruppen ergaben } \\
\text { sich im Ausgangs-MLD }(0,92 \pm 0,34 \mathrm{~mm} \text { vs. } \\
0,85 \pm 0,36 \mathrm{~mm}) \text {, der Läsionslänge }(13,6 \\
\pm 6,11 \mathrm{~mm} \text { vs. } 14,76 \pm 6,61) \text {, dem Ausmaß } \\
\text { der Gefäßschlängelung }(8,4 \text { vs. } 13,1 \%) \\
\text { und dem Anteil an verkalkten Gefäße }(23,1 \\
\text { vs. } 29,8 \%) \text {. }\end{array}$ \\
\hline
\end{tabular}

\section{Prozedurdaten}

Prozedurzeit

Anzahl der Postdilatations-

Ballonkatheter

Stentlänge/Läsionslänge

Bailout stenting

Geographic miss

Primärer Endpunkt

TVR nach 9 Monaten

TLR

TVR remote

MACE

Stentthrombose

Tod

$\begin{array}{cc}53,0 \pm 49,5 \mathrm{~min} & 47,8 \pm 25,5 \mathrm{~min}^{*} \\ 1,3 \pm 0,9 & 1,1 \pm 0,4^{*} \\ 1,8 \pm 0,9 & 1,7 \pm 0,7^{*} \\ 6,0 \% & 3,1 \%{ }^{*} \\ 9,2 \% & 5,6 \%^{*} \\ & \\ 7,1 \% & 8,0 \% \\ 4,5 \% & 5,7 \% \\ 2,7 \% & 3,2 \% \\ 10,5 \% & 11,0 \% \\ 0,7 \% & 0,8 \% \\ 1,8 \% & 1,2 \% \\ 0,42 \pm 0,54 \mathrm{~mm} & 0,41 \pm 0,54 \mathrm{~mm}\end{array}$

waren (s. o.). Das klinische und angiographische Ergebnis war vergleichbar, so dass der TAXUS-Liberté-Stent als adäquater bzw. verbesserter Ersatz des TAXUS-Express-Stents angesehen werden kann. Ein solches Studiendesign wäre zur Prüfung aller ,kleineren“ Modifikationen von Koronarstents wünschenswert.

S. Silber, München

Herz 2006;31:472

DOI 10.1007/s00059-006-2878-7 